\title{
The Effect of Uncertainty on Investment Timing in a Real Options Model*
}

\author{
Kit Pong WONG ${ }^{\dagger}$ \\ University of Hong Kong
}

July 2006

\begin{abstract}
This paper examines the effect of uncertainty on investment timing in a canonical real options model. We show that the critical value of a project that triggers the exercise of the investment option exhibits a U-shaped pattern against the volatility of the project. This is due to the two countervailing risk and return factors in effect. We further show that such a U-shaped pattern is inherited by the expected time to exercise the investment option. Thus, for relatively safe projects, greater uncertainty shortens the expected exercise time and thus enhances investment. This is in sharp contrast to the negative investmentuncertainty relationship commonly found in the extant literature. Finally, we show that the positive investment-uncertainty relationship is more likely for high growth projects than for low growth projects.
\end{abstract}

JEL classification: D21; D81; G13

Keywords: Real options; Investment timing; Uncertainty

\footnotetext{
${ }^{*}$ I gratefully acknowledge financial support by a grant from the University Grants Committee of the Hong Kong Special Administrative Region, China (Project No. AoE/H-05/99). I would like to thank Carl Chiarella (the editor), Rujing Meng, Sudipto Sarkar, and an anonymous referee for their helpful comments and suggestions, and Ho Yin Yick for his excellent research assistance. Any remaining errors are of course mine.

${ }^{\dagger}$ Correspondence to: Kit Pong Wong, School of Economics and Finance, University of Hong Kong, Pokfulam Road, Hong Kong. Tel.: (852) 2859-1044, fax: (852) 2548-1152, e-mail: kpwong@econ.hku.hk (K. P. Wong).
} 


\title{
The effect of uncertainty on investment timing in a real options model
}

\author{
Kit Pong Wong* \\ School of Economics and Finance, University of Hong Kong, Pokfulam Road, Hong Kong
}

\begin{abstract}
This paper examines the effect of uncertainty on investment timing in a canonical real options model. We show that the critical value of a project that triggers the exercise of the investment option exhibits a U-shaped pattern against the volatility of the project. This is due to the two countervailing risk and return factors in effect. We further show that such a U-shaped pattern is inherited by the expected time to exercise the investment option. Thus, for relatively safe projects, greater uncertainty shortens the expected exercise time and thus enhances investment. This is in sharp contrast to the negative investment-uncertainty relationship commonly found in the extant literature. Finally, we show that the positive investment-uncertainty relationship is more likely for high growth projects than for low growth projects.
\end{abstract}

JEL classification: D21; D81; G13

Keywords: Real options; Investment timing; Uncertainty

\section{Introduction}

Economists have long been fascinated by the investment-uncertainty relationship. The extant literature by and large dictates a negative sign to such a relationship (see Caballero, 1991; Leahy and Whited, 1996). Using a canonical real options model of McDonald and Siegel (1986) and Dixit and Pindyck (1994), Sarkar (2000) shows that the investmentuncertainty relationship is not necessarily monotonic. Viewing investment opportunities

${ }^{*}$ Tel.: +852-2859-1044; fax: +852-2548-1152.

E-mail address: kpwong@econ.hku.hk (K.P. Wong). 
as perpetual American call options, firms endogenously devise their investment timing so as to maximize the option values. The optimal decision rule is that a project should be undertaken at the moment when the value of the project reaches a critical level (the optimal investment trigger). To gauge the effect of uncertainty on investment, Sarkar (2000) uses the probability of reaching the optimal investment trigger within a prespecified period of time. If this probability measure increases (decreases) with the volatility of the project, one can reasonably infer a positive (negative) sign of the uncertainty-investment relationship.

This paper follows the real options approach of Sarkar (2000), albeit with a caveat. In order to draw implications regarding the investment-uncertainty relationship, we use the effect of uncertainty on investment timing rather than on the probability measure as suggested by Sarkar (2000). Investment timing is defined by the expected time to reach the optimal investment trigger (see also Shackleton and Wojakowski, 2002; Grenadier and Wang, 2005). Given that the value of the project evolves over time according to a geometric Brownian motion, the expected exercise time takes on a much simpler functional form than the probability measure used by Sarkar (2000) (see Karlin and Taylor, 1975). This offers great advantage to us that clean analytical results can be easily derived and clearly explained.

As in Sarkar (2000), we employ the single-factor intertemporal capital asset pricing model (CAPM) of Merton (1973a) to determine the risk-adjusted rate of return on the project. In the real options literature, it is generally believed that the optimal investment trigger is an increasing function of the underlying volatility of the project. ${ }^{1}$ Dixit and Pindyck (1994) verify such a comparative statics result by implicitly assuming that the riskadjusted rate of return on the project is invariant to the volatility, which contradicts the CAPM in that a linearly positive relationship between risk and return exists in equilibrium. We show that the behavior of the optimal investment trigger with respect to the volatility of the project is non-monotonic. There are two countervailing factors, the risk factor and the return factor, in effect. When the volatility of the project goes up, the risk factor enhances

\footnotetext{
${ }^{1}$ For example, Sarkar (2000) states that "it is obvious that a higher level of uncertainty will increase the critical trigger level ..., and thereby have a negative effect on investment (p. 222)."
} 
the value of the option to invest in the project and thus makes waiting more beneficial. This lifts up the exercise trigger. On the other hand, the return factor arising from the upward adjustment of the discount rate makes waiting more costly. This pushes down the exercise trigger. The return factor dominates for low levels of uncertainty, while the risk factor dominates for high levels of uncertainty, thereby rendering a U-shaped pattern of the optimal investment trigger against the volatility of the project.

We further show that the expected exercise time inherits the U-shaped pattern of the optimal investment trigger against the volatility of the project. Specifically, the return factor that calls for shortening the exercise time dominates for relatively safe projects, while the risk factor that calls for lengthening the exercise time dominates for sufficiently risky projects. Thus, it is quite possible that greater uncertainty may in fact lure firms into making more investment through shortening the expected exercise time, especially when projects are relatively safe. This is in sharp contrast to the negative investment-uncertainty relationship commonly found in the extant literature. Finally, we show that the positive investment-uncertainty relationship is more likely to prevail for high growth projects than for low growth projects.

The rest of this paper is organized as follows. The next section delineates the model and characterizes the optimal investment trigger. Sections 3 and 4 examine how the optimal investment trigger and the investment timing respond to increased volatility of the project, respectively. Section 5 offers a numerical example to gain additional insight. The final section concludes.

\section{The model}

Consider a canonical real options model à la McDonald and Siegel (1986) and Dixit and Pindyck (1994). In this model, a firm has an option to invest in a project whose value, 
$V(t)$, evolves over time according to the following geometric Brownian motion:

$$
\mathrm{d} V(t)=\alpha V(t) \mathrm{d} t+\sigma V(t) \mathrm{d} W(t)
$$

where $W(t)$ is a standard Wiener process, and $\alpha$ and $\sigma$ are the drift rate (expected growth rate) and volatility (standard derivation) per unit of time, respectively, with $\alpha>\sigma^{2} / 2 .^{2}$ The firm can undertake the project at any time by paying a deterministic investment cost, $I$, at that moment. The initial value of the project is denoted by $V_{0}=V(0)$.

As in Sarkar (2000), we assume that the risk-adjusted rate of return on the project is determined by the single-factor intertemporal capital asset pricing model (CAPM) of Merton (1973a). The per-unit value of the market portfolio, $P_{m}(t)$, is governed by the following geometric Brownian motion:

$$
\mathrm{d} P_{m}(t)=\mu_{m} P_{m}(t) \mathrm{d} t+\sigma_{m} P_{m}(t) \mathrm{d} Z_{m}(t)
$$

where $Z_{m}(t)$ is a standard Wiener process correlated with $W(t)$, and $\mu_{m}$ and $\sigma_{m}$ are the drift rate and volatility per unit of time, respectively. Denote $r$ as the riskless rate of interest and $\lambda=\left(\mu_{m}-r\right) / \sigma_{m}$ as the market price of risk.

Alternatively, we can write Eq. (1) as

$$
\mathrm{d} V(t)=\alpha V(t) \mathrm{d} t+\rho \sigma V(t) \mathrm{d} Z_{m}(t)+\sqrt{1-\rho^{2}} \sigma V(t) \mathrm{d} Z(t)
$$

where $Z(t)$ is a standard Wiener process independent of $Z_{m}(t)$, and $\rho \in[-1,1]$ is the correlation coefficient between the value of the project and the return on the market portfolio. Inspection of Eq. (3) reveals that $\rho^{2} \sigma^{2}$ and $\left(1-\rho^{2}\right) \sigma^{2}$ capture the systematic risk and the idiosyncratic risk of the project, respectively.

According to the CAPM, the risk-adjusted rate of return that investors would require if they are to own the project is given by $r+\lambda \rho \sigma$. Let $F\left(V_{0}\right)$ be the value of the option to

\footnotetext{
${ }^{2}$ Using Ito's Lemma, Eq. (1) implies that $\ln V(t)$ follows a Brownian motion with drift $\alpha-\sigma^{2} / 2$ and volatility $\sigma$. The assumption that $\alpha>\sigma^{2} / 2$ ensures that the drift rate is positive. As is evident from Eq. (22), this assumption is necessary for a meaningful expected time to exercise the investment option. If we follow Sarkar (2000) to use the probability of investment rather than the expected time to investment, we no longer require such an assumption.
} 
invest in the project (hereafter referred to as the investment option), evaluated at time 0. Since the payoff from undertaking the project at time $t$ is given by $V(t)-I$, we have

$$
F\left(V_{0}\right)=\max _{t^{*}} \mathrm{E}\left\{\left[V\left(t^{*}\right)-I\right] e^{-(r+\lambda \rho \sigma) t^{*}}\right\}
$$

subject to Eq. (1). To find the optimal time, $t^{*}$, at which the investment option is exercised is tantamount to solving the critical value of the project, $V^{*}$, that triggers the exercise of the investment option. To ensure that $V^{*}$ is finite, we assume that $\alpha<r+\lambda \rho \sigma{ }^{3}$

Using the standard argument (see Appendix A), the value of the investment option at time $t, F[V(t)]$, satisfies the following differential equation:

$$
\frac{1}{2} \sigma^{2} V(t)^{2} F^{\prime \prime}[V(t)]+(\alpha-\lambda \rho \sigma) V(t) F^{\prime}[V(t)]-r F[V(t)]=0,
$$

subject to the following boundary conditions:

$$
\begin{aligned}
& F(0)=0, \\
& F\left(V^{*}\right)=V^{*}-I, \\
& F^{\prime}\left(V^{*}\right)=1 .
\end{aligned}
$$

Eq. (6) reflects the fact that zero is an absorbing barrier for the geometric Brownian motion in Eq. (1). Eq. (7) is the value-matching condition such that at the moment the investment option is exercised, its payoff is equal to the net present value of the project. Eq. (8) is the smooth-pasting condition such that the optimal investment trigger is the one that maximizes the value of the investment option (Dumas, 1991; Dixit, 1993). ${ }^{4}$

Eq. (5) is a second-order linear homogeneous differential equation. The general solution takes the form of a power function, $A V^{\beta}$, where $A$ is a constant to be determined and $\beta$ is a solution to the following quadratic equation:

$$
\frac{1}{2} \sigma^{2} \beta(\beta-1)+(\alpha-\lambda \rho \sigma) \beta-r=0 .
$$

\footnotetext{
${ }^{3}$ For this convergence issue, see McDonald and Siegel (1986) and Dixit and Pindyck (1994). See also Eq. (11).

${ }^{4}$ Shackleton and Sødal (2005) show that smooth pasting implies rate of return equalization between the option and the levered position that results from exercise.
} 
There are two roots for Eq. (9), one positive and the other negative. For Eq. (6) to hold, the coefficient for the negative $\beta$ must be zero and therefore we have

$$
\beta=\frac{1}{2}-\frac{\alpha-\lambda \rho \sigma}{\sigma^{2}}+\sqrt{\left(\frac{1}{2}-\frac{\alpha-\lambda \rho \sigma}{\sigma^{2}}\right)^{2}+\frac{2 r}{\sigma^{2}}} .
$$

Given that $\alpha<r+\lambda \rho \sigma$, it is evident from Eq. (10) that $\beta>1$.

Using $F(V)=A V^{\beta}$ to solve Eqs. (7) and (8), we derive the optimal investment trigger as

$$
V^{*}=\frac{\beta}{\beta-1} I
$$

and the value of the investment option at time 0 as

$$
F\left(V_{0}\right)= \begin{cases}\left(V^{*}-I\right)\left(V_{0} / V^{*}\right)^{\beta} & \text { if } V_{0}<V^{*} \\ V_{0}-I & \text { if } V_{0} \geq V^{*}\end{cases}
$$

where $\left(V_{0} / V^{*}\right)^{\beta}$ is the stochastic discount factor that accounts for both the timing and the probability of one dollar received at the first moment when the optimal investment trigger, $V^{*}$, is reached. To ensure some positive option value inherent in the project, in the sequel we assume that $V_{0}<V^{*}$.

\section{The trigger-uncertainty relationship}

In this section, we study the effect of uncertainty on the optimal investment trigger, $V^{*}$. Sarkar (2000) refers to increased uncertainty as an increase in $\sigma$, taking all other parameters, $r, \lambda, \rho$, and $\alpha$, as constants. In this case, the increase in $\sigma$ has a systematic risk component and thus affects the dividend stream, $r+\lambda \rho \sigma-\alpha$, in accord with the CAPM. On the other hand, McDonald and Siegel (1986) and Dixit and Pindyck (1994) consider another type of increased uncertainty in which the dividend stream is held fixed when $\sigma$ changes. In 
this case, the increase in $\sigma$ has only an idiosyncratic risk component. ${ }^{5}$ While we follow the approach of Sarkar (2000), we can also get the scenario of McDonald and Siegel (1986) and Dixit and Pindyck (1994) by setting $\rho=0$.

Differentiating Eq. (9) with respect to $\sigma$ yields

$$
\frac{\partial \beta}{\partial \sigma}=\frac{\beta[\lambda \rho-\sigma(\beta-1)]}{\sigma^{2}(\beta-1 / 2)+\alpha-\lambda \rho \sigma}
$$

Differentiating Eq. (11) with respect to $\sigma$ yields

$$
\frac{\partial V^{*}}{\partial \sigma}=-\frac{I}{(\beta-1)^{2}} \frac{\partial \beta}{\partial \sigma}=\frac{V^{*}[\sigma(\beta-1)-\lambda \rho]}{(\beta-1)\left[\sigma^{2}(\beta-1 / 2)+\alpha-\lambda \rho \sigma\right]}
$$

where the second equality follows from Eq. (13). If $\rho \leq 0$, it is evident from Eq. (14) that $\partial V^{*} / \partial \sigma>0$ for all $\sigma \in(0, \sqrt{2 \alpha})$. However, if $\rho>0$, the trigger-uncertainty relationship is no longer monotonic, as is shown in the following proposition.

Proposition 1. If $\rho \leq 0, \partial V^{*} / \partial \sigma>0$ for all $\sigma \in(0, \sqrt{2 \alpha})$. If $\rho>0$, there exists a unique point, $\sigma^{*} \in(0, \sqrt{2 \alpha})$, defined by

$$
\sigma^{*}=\sqrt{\left(\frac{2 r-2 \alpha+\lambda^{2} \rho^{2}}{2 \lambda \rho}\right)^{2}+2 \alpha}-\frac{2 r-2 \alpha+\lambda^{2} \rho^{2}}{2 \lambda \rho}
$$

such that $\partial V^{*} / \partial \sigma<0$ for all $\sigma \in\left(0, \sigma^{*}\right)$ and $\partial V^{*} / \partial \sigma>0$ for all $\sigma \in\left(\sigma^{*}, \sqrt{2 \alpha}\right)$.

Proof. See Appendix B.

In the real options literature, it is generally believed that the optimal investment trigger, $V^{*}$, is strictly increasing in the underlying volatility, $\sigma$. Dixit and Pindyck (1994, p. 144) verify such a comparative statics result by implicitly assuming that the risk-adjusted rate of return on the project is invariant to $\sigma$. Of course, this assumption is innocuous if the increase in $\sigma$ is solely idiosyncratic in nature, which is qualitatively identical to our case when $\rho=0$.

\footnotetext{
${ }^{5}$ See also Cappuccio and Moretto (2001) and Lund (2005) for the distinction between these two types of increased uncertainty.
} 
According to the CAPM, the risk-adjusted rate of return on the project is linearly related to $\sigma$ in general, and such a relationship is positive when $\rho>0$ in particular. It is this positive relationship between risk and return, which has not been properly accounted for in the real options literature, that drives the U-shaped pattern of the optimal investment trigger against the volatility in the case that $\rho>0$.

To see the intuition, let us consider the hypothetical case without any uncertainty. When $\sigma=0$, we have $V(t)=V_{0} e^{\alpha t}$. Program (4) becomes

$$
F\left(V_{0}\right)=\max _{t^{*}}\left[V_{0} e^{\alpha t^{*}}-I\right] e^{-\mu t^{*}}
$$

where $\mu$ is the discount rate and $\mu>\alpha$ to ensure convergence. As shown in Dixit and Pindyck (1994, p. 138), the exercise trigger that solves program (16) is given by

$$
V^{*}=\frac{\mu}{\mu-\alpha} I
$$

if $V^{*}>V_{0}$. Differentiating Eq. (17) with respect to $\mu$ yields

$$
\frac{\partial V^{*}}{\partial \mu}=-\frac{\alpha}{(\mu-\alpha)^{2}} I<0
$$

In words, an increase in the discount rate makes waiting more costly and thus induces the firm to lower the optimal investment trigger in the deterministic case.

In the case of uncertainty, two factors are in effect when the volatility of the project goes up. To see this, we rewrite Eq. (14) as

$$
\frac{\partial V^{*}}{\partial \sigma}=\frac{V^{*} \sigma}{\sigma^{2}(\beta-1 / 2)+\alpha-\lambda \rho \sigma}-\frac{V^{*} \lambda \rho}{(\beta-1)\left[\sigma^{2}(\beta-1 / 2)+\alpha-\lambda \rho \sigma\right]} .
$$

The first term on the right-hand side of Eq. (19) captures the risk factor that holds the riskadjusted rate of return on the project constant at $r+\lambda \rho \sigma$. The risk factor is unambiguously positive because an increase in $\sigma$ enhances the value of the investment option (Merton, 1973b) and thus induces the firm to wait longer by lifting up the exercise trigger. The second term on the right-hand side of Eq. (19) captures the return factor that holds the value of the investment option constant at $\sigma$. The return factor is positive or negative, 
depending on whether $\rho$ is negative or positive, respectively. According to the CAPM, an increase in $\sigma$ renders the discount rate to be adjusted upward (downward) if $\rho>(<) 0$. As shown in Eq. (18), waiting becomes more (less) costly and thus the firm is induced to lower (raise) the exercise trigger.

If $\rho<0$, the risk and return factors reinforce each other. The trigger-uncertainty relationship is positive in line with the conventional wisdom in the extant literature. On the other hand, if $\rho>0$, the two factors act against each other. When there is relatively little uncertainty, it is evident from Eq. (19) that the risk factor has at best second-order significance while the return factor always has first-order effect because the risk-adjusted rate of return on the project is linear in $\sigma$ in accord with the CAPM. Thus, for all $\sigma<(>) \sigma^{*}$, the return factor dominates (is dominated by) the risk factor, thereby making the optimal investment trigger exhibit a U-shaped pattern against the volatility of the project.

\section{The investment-uncertainty relationship}

In this section, we study the effect of uncertainty on the expected time to invest. Let $T$ be the first passage time that the value of the project reaches the optimal investment trigger, $V^{*}$, from the initial value, $V_{0}$. Using Ito's Lemma, Eq. (1) implies that $\ln V(t)$ follows a Brownian motion with drift $\alpha-\sigma^{2} / 2$ and volatility $\sigma$. Applying Theorem 5.3 in Karlin and Taylor (1975, p. 363), the probability density function of $T$ is given by

$$
\phi(T)=\frac{\ln \left(V^{*} / V_{0}\right)}{\sigma \sqrt{2 \pi T^{3}}} e^{-\frac{\left[\ln \left(V^{*} / V_{0}\right)-\left(\alpha-\sigma^{2} / 2\right) T\right]}{2 \sigma^{2} T}},
$$

and the Laplace transform of $T$ is given by

$$
\mathrm{E}\left(e^{-\theta T}\right)=\int_{0}^{\infty} e^{-\theta T} \phi(T) \mathrm{d} T=e^{-\left[\sqrt{\left(\alpha-\sigma^{2} / 2\right)^{2}+2 \sigma^{2} \theta}-\left(\alpha-\sigma^{2} / 2\right)\right] \ln \left(V^{*} / V_{0}\right) / \sigma^{2}}
$$

where $\mathrm{E}(\cdot)$ is the expectation operator with respect to $\phi(T)$ defined in Eq. (20). 
Based on Eq. (21), we can derive the expected time to exercise the investment option as (see also Shackleton and Wojakowski, 2002; Grenadier and Wang, 2005)

$$
\mathrm{E}(T)=\int_{0}^{\infty} T \phi(T) \mathrm{d} T=-\lim _{\theta \rightarrow 0} \frac{\partial \mathrm{E}\left(e^{-\theta T}\right)}{\partial \theta}=\frac{\ln \left(V^{*} / V_{0}\right)}{\alpha-\sigma^{2} / 2}
$$

Differentiating Eq. (22) with respect to $\sigma$ yields

$$
\frac{\partial \mathrm{E}(T)}{\partial \sigma}=\frac{1}{\left(\alpha-\sigma^{2} / 2\right)^{2}}\left[\sigma \ln \left(\frac{V^{*}}{V_{0}}\right)+\left(\alpha-\frac{\sigma^{2}}{2}\right) \frac{1}{V^{*}} \frac{\partial V^{*}}{\partial \sigma}\right]
$$

If $\rho \leq 0$, it is evident from Proposition 1 and Eq. (23) that $\partial \mathrm{E}(T) / \partial \sigma>0$ for all $\sigma \in$ $(0, \sqrt{2 \alpha})$. However, if $\rho>0$, Proposition 1 says that the trigger-uncertainty relationship is U-shaped. As is shown in the following proposition, the investment-uncertainty relationship is also U-shaped in the case that $\rho>0$.

Proposition 2. If $\rho \leq 0$, we have $\partial \mathrm{E}(T) / \partial \sigma>0$ for all $\sigma>0$. If $\rho>0$, there exists a unique point, $\sigma^{\mathrm{o}} \in\left(0, \sigma^{*}\right)$, implicitly defined by

$$
\left.\frac{\partial \mathrm{E}(T)}{\partial \sigma}\right|_{\sigma=\sigma^{\circ}}=0
$$

such that $\partial \mathrm{E}(T) / \partial \sigma<0$ for all $\sigma \in\left(0, \sigma^{\circ}\right)$ and $\partial \mathrm{E}(T) / \partial \sigma>0$ for all $\sigma \in\left(\sigma^{\circ}, \sqrt{2 \alpha}\right)$.

Proof. See Appendix C.

To see the intuition of Proposition 2, we use Eq. (19) to recast Eq. (23) as

$$
\begin{aligned}
\frac{\partial \mathrm{E}(T)}{\partial \sigma}= & \sigma\left\{\frac{\ln \left(V^{*} / V_{0}\right)}{\left(\alpha-\sigma^{2} / 2\right)^{2}}+\frac{1}{\left(\alpha-\sigma^{2} / 2\right)\left[\sigma^{2}(\beta-1 / 2)+\alpha-\lambda \rho \sigma\right]}\right\} \\
& -\frac{\lambda \rho}{\left(\alpha-\sigma^{2} / 2\right)(\beta-1)\left[\sigma^{2}(\beta-1 / 2)+\alpha-\lambda \rho \sigma\right]}
\end{aligned}
$$

Inspection of Eq. (25) reveals two factors that govern the expected time to invest when the volatility of the project goes up. As in the previous section, the first term on the 
right-hand side of Eq. (25) captures the risk factor while the second term captures the return factor. If $\rho<0$, the two factors reinforce each other so that the firm cuts down its investment by stretching the expected exercise time in response to increased uncertainty. In the more plausible case that $\rho>0$, Proposition 2 states that greater uncertainty may in fact lure the firm into making more investment through shortening the expected exercise time, especially when the project is relatively safe (i.e., $\sigma<\sigma^{\circ}$ ). When the project is sufficiently risky (i.e., $\sigma>\sigma^{\circ}$ ), the usual negative investment-uncertainty relationship as suggested in the extant literature prevails. This non-monotonic investment-uncertainty relationship is driven by the U-shaped pattern of the optimal investment trigger against the volatility of the project. Specifically, the return factor that calls for shortening the exercise time dominates for relatively safe projects, while the risk factor that calls for lengthening the exercise time dominates for sufficiently risky projects.

Finally, it is of interest to examine whether the positive investment-uncertainty relationship is more likely to be observed among high growth projects or among low growth projects. To this end, we conduct a comparative statics exercise of $\sigma^{\circ}$ with respect to $\alpha$ in the following proposition.

Proposition 3. Consider the case that $\rho>0$. If the following condition holds:

$$
\ln \left(\frac{r}{r-\alpha}\right)+\ln \left(\frac{I}{V_{0}}\right) \geq \frac{\alpha}{r-\alpha}
$$

then $\mathrm{d} \sigma^{\mathrm{o}} / \mathrm{d} \alpha>0$.

Proof. See Appendix D.

The intuition of Proposition 3 is as follows. In the deterministic case, we can use Eq. (18) to derive

$$
\frac{\partial^{2} V^{*}}{\partial \mu \partial \alpha}=-\frac{\mu+\alpha}{(\mu-\alpha)^{3}} I<0 .
$$


In words, Eq. (27) implies that, as the growth rate of the project increases, the firm under certainty is induced to lower the optimal investment trigger further in response to an increase in the discount rate. The return factor is thus stronger for high growth projects than for low growth projects, ceteris paribus. The effect of a higher growth rate of the project on the risk factor, however, is indeterminate. Condition (26) offers a sufficient (but not necessary) condition for the positive effect on the return factor to dominate so that $\mathrm{d} \sigma^{\mathrm{o}} / \mathrm{d} \alpha>0$. Condition (26) holds when the initial net present value of the project, $V_{0}-I$, is sufficiently negative, or when the wedge between the riskless rate of interest and the growth rate of the project, $r-\alpha$, is sufficiently large. In this case, the positive investment-uncertainty relationship is more likely for high growth projects than for low growth projects.

\section{Numerical analysis}

To gain more insight, we construct a numerical example in this section. As in Sarkar (2000), we use the following parameter values: $r=0.1, \lambda=0.4, \rho=0.7, V_{0}=0.1$, and $I=1$. We employ Eq. (22) to compute the expected exercise time, $\mathrm{E}(T)$, for different values of $\sigma \in(0, \sqrt{2 \alpha})$ and $\alpha \in\{0.03,0.05,0.07\} .{ }^{6}$ Fig. 1 depicts the behavior of $\mathrm{E}(T)$ with respect to $\sigma$ for the three different values of $\alpha$.

\footnotetext{
${ }^{6}$ The choice of the parameter values of $r, \alpha, V_{0}$, and $I$ satisfies condition (26).
} 


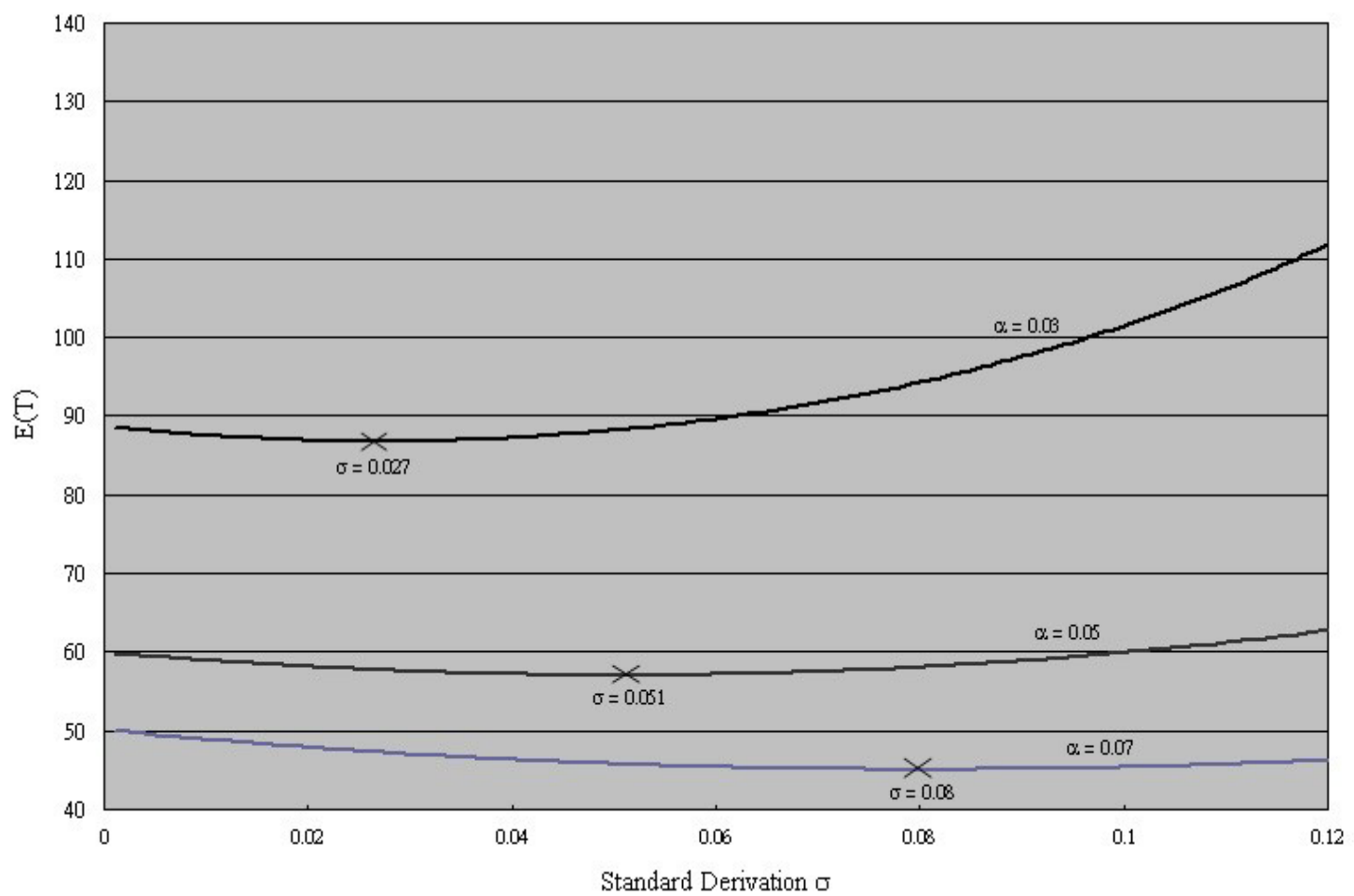

Fig. 1. The relationship between the expected exercise time, $\mathrm{E}(T)$, and the volatility of the project, $\sigma$, when $r=0.1, \lambda=0.4, \rho=0.7, V_{0}=0.1$, and $I=1$.

As is evident from Fig. 1, the expected exercise time first decreases and then increases with $\sigma$. The turning points are at $\sigma=0.08$ when $\alpha=0.07, \sigma=0.051$ when $\alpha=0.05$, and $\sigma=0.027$ when $\alpha=0.03$. For $\sigma$ sufficiently low, an increase in $\sigma$ shortens the expected time to exercise the investment option and thereby has a positive effect on the level of investment. The numerical example also shows that such a positive investment-uncertainty relationship is more likely to be observed for high growth projects than for low growth projects.

\section{Conclusions}

This paper has examined the effect of uncertainty on investment timing in a canonical 
real options model of McDonald and Siegel (1986) and Dixit and Pindyck (1994). Specifically, we have shown that the critical value of a project (the optimal investment trigger) that triggers the exercise of the investment option exhibits a U-shaped pattern against the volatility of the project. This U-shaped pattern is due to two countervailing factors in effect. When the volatility of the project goes up, the risk factor enhances the value of the investment option and thus makes waiting more beneficial. This lifts up the exercise trigger. On the other hand, the return factor arising from the upward adjustment of the discount rate makes waiting more costly. This pushes down the exercise trigger. The return factor dominates for low levels of uncertainty, while the risk factor dominates for high levels of uncertainty. We have further shown that the U-shaped pattern of the optimal investment trigger against the volatility is inherited by the expected time to exercise the investment option. Thus, for relatively safe projects, greater uncertainty may in fact shorten the expected exercise time and thereby lure firms into making more investment. This is in sharp contrast to the usual negative investment-uncertainty relationship as suggested in the extant literature. Finally, we have shown that the positive investment-uncertainty relationship is more likely for high growth projects than for low growth projects.

\section{Acknowledgements}

I gratefully acknowledge financial support by a grant from the University Grants Committee of the Hong Kong Special Administrative Region, China (Project No. AoE/H05/99). I would like to thank Carl Chiarella (the editor), Rujing Meng, Sudipto Sarkar, and an anonymous referee for their helpful comments and suggestions, and Ho Yin Yick for his excellent research assistance. Any remaining errors are of course mine. 
Appendix A. Derivation of Eq. (5)

Consider the following dynamic portfolio at time $t$ : (i) Hold the investment option that is worth $F[V(t)]$, and (ii) go short $n$ units of the market portfolio. The value of this portfolio is $F[V(t)]-n P_{m}(t)$. The total return from holding the portfolio over a short time interval, $\mathrm{d} t$, is

$$
\begin{aligned}
& \mathrm{d} F[V(t)]-n \mathrm{~d} P_{m}(t) \\
& =F^{\prime}[V(t)] \mathrm{d} V(t)+\frac{1}{2} F^{\prime \prime}[V(t)][\mathrm{d} V(t)]^{2}-n \mu_{m} P_{m}(t) \mathrm{d} t-n \sigma_{m} P_{m}(t) \mathrm{d} Z_{m}(t) \\
& =\left\{\alpha V(t) F^{\prime}[V(t)]+\frac{1}{2} \sigma^{2} V(t)^{2} F^{\prime \prime}[V(t)]-n \mu_{m} P_{m}(t)\right\} \mathrm{d} t \\
& \quad+\left\{\rho \sigma V(t) F^{\prime}[V(t)]-n \sigma_{m} P_{m}(t)\right\} \mathrm{d} Z_{m}(t)+\sqrt{1-\rho^{2}} \sigma V(t) F^{\prime}[V(t)] \mathrm{d} Z(t),
\end{aligned}
$$

where the first equality follows from Ito's Lemma, and the second equality follows from Eq. (3) and $[\mathrm{d} V(t)]^{2}=\sigma^{2} V(t)^{2} \mathrm{~d} t$. Substituting $n=\rho \sigma V(t) F^{\prime}[V(t)] / \sigma_{m} P_{m}(t)$ into Eq. (A.1) yields

$$
\begin{aligned}
& \mathrm{d} F[V(t)]-\frac{\rho \sigma V(t) F^{\prime}[V(t)]}{\sigma_{m} P_{m}(t)} \mathrm{d} P_{m}(t) \\
& =\left\{\left[\alpha-\frac{\mu_{m} \rho \sigma}{\sigma_{m}}\right] V(t) F^{\prime}[V(t)]+\frac{1}{2} \sigma^{2} V(t)^{2} F^{\prime \prime}[V(t)]\right\} \mathrm{d} t \\
& \quad+\sqrt{1-\rho^{2}} \sigma V(t) F^{\prime}[V(t)] \mathrm{d} Z(t) .
\end{aligned}
$$

Inspection of Eq. (A.2) reveals that the only risk associated with the dynamic portfolio over $\mathrm{d} t$ is diversifiable and therefore the expected rate of return on the portfolio must be equal to the riskless rate of interest, $r$. To avoid arbitrage opportunities, we have

$$
\left\{\left[\alpha-\frac{\mu_{m} \rho \sigma}{\sigma_{m}}\right] V(t) F^{\prime}[V(t)]+\frac{1}{2} \sigma^{2} V(t)^{2} F^{\prime \prime}[V(t)]\right\} \mathrm{d} t
$$




$$
=r\left\{F[V(t)]-\frac{\rho \sigma}{\sigma_{m}} V(t) F^{\prime}[V(t)]\right\} \mathrm{d} t
$$

Eliminating $\mathrm{d} t$ on both sides of Eq. (A.3) and rearranging terms yields Eq. (5).

\section{Appendix B. Proof of Proposition 1}

Using Eq. (10), we have

$$
\sigma^{2}\left(\beta-\frac{1}{2}\right)+\alpha-\lambda \rho \sigma=\sigma \sqrt{\left(\frac{\sigma}{2}-\frac{\alpha-\lambda \rho \sigma}{\sigma}\right)^{2}+2 r}>0
$$

Eqs. (14) and (A.4) imply that $\partial V^{*} / \partial \sigma$ has the same sign as that of $\sigma(\beta-1)-\lambda \rho$, which is negative if $\rho \leq 0$ and ambiguous if $\rho>0$.

For the case that $\rho>0$, we write Eq. (10) as

$$
\sigma(\beta-1)-\lambda \rho=\sqrt{\left(\frac{\sigma}{2}-\frac{\alpha-\lambda \rho \sigma}{\sigma}\right)^{2}+2 r}-\frac{2 \alpha+\sigma^{2}}{2 \sigma} .
$$

Note that

$$
\begin{aligned}
& {\left[\sqrt{\left(\frac{\sigma}{2}-\frac{\alpha-\lambda \rho \sigma}{\sigma}\right)^{2}+2 r}-\frac{2 \alpha+\sigma^{2}}{2 \sigma}\right]\left[\sqrt{\left(\frac{\sigma}{2}-\frac{\alpha-\lambda \rho \sigma}{\sigma}\right)^{2}+2 r}+\frac{2 \alpha+\sigma^{2}}{2 \sigma}\right]} \\
& =\left(\frac{\sigma}{2}-\frac{\alpha-\lambda \rho \sigma}{\sigma}\right)^{2}+2 r-\left(\frac{2 \alpha+\sigma^{2}}{2 \sigma}\right)^{2} \\
& =\frac{1}{\sigma}\left[\lambda \rho \sigma^{2}+\left(2 r-2 \alpha+\lambda^{2} \rho^{2}\right) \sigma-2 \alpha \lambda \rho\right] .
\end{aligned}
$$

Inspection of Eqs. (A.5) and (A.6) reveals that $\sigma(\beta-1)-\lambda \rho$ has the same sign as that of $\lambda \rho \sigma^{2}+\left(2 r-2 \alpha+\lambda^{2} \rho^{2}\right) \sigma-2 \alpha \lambda \rho$, which is negative or positive depending on whether $\sigma$ is lower or higher than $\sigma^{*}$, respectively, where $\sigma^{*}$ is defined in Eq. (15). Note that

$$
\left(\frac{2 r-2 \alpha+\lambda^{2} \rho^{2}}{2 \lambda \rho}\right)^{2}+2 \alpha<\left(\frac{2 r-2 \alpha+\lambda^{2} \rho^{2}}{2 \lambda \rho}+\sqrt{2 \alpha}\right)^{2}
$$


It then follows from Eq. (15) and inequality (A.7) that $\sigma^{*}<\sqrt{2 \alpha}$.

\section{Appendix C. Proof of Proposition 2}

If $\rho \leq 0$, we know from Proposition 1 that $\partial V^{*} / \partial \sigma>0$ for all $\sigma \in(0, \sqrt{2 \alpha})$. Thus, it follows from Eq. (23) that $\partial \mathrm{E}(T) / \partial \sigma>0$ for all $\sigma \in(0, \sqrt{2 \alpha})$. On the other hand, if $\rho>0$, Proposition 1 implies that $\partial V^{*} / \partial \sigma<0$ for all $\sigma \in\left(0, \sigma^{*}\right)$ and $\partial V^{*} / \partial \sigma>0$ for all $\sigma \in\left(\sigma^{*}, \sqrt{2 \alpha}\right)$. It then follows from Eq. (23) that $\partial \mathrm{E}(T) / \partial \sigma>0$ for all $\sigma \in\left[\sigma^{*}, \sqrt{2 \alpha}\right)$ but has an ambiguous sign for all $\sigma \in\left(0, \sigma^{*}\right)$.

For the case that $\rho>0$, define the expression inside the squared brackets on the righthand side of Eq. (23) as $M$ :

$$
M=\sigma \ln \left(\frac{V^{*}}{V_{0}}\right)+\left(\alpha-\frac{\sigma^{2}}{2}\right) \frac{1}{V^{*}} \frac{\partial V^{*}}{\partial \sigma} .
$$

Differentiating Eq. (A.8) with respect to $\sigma$ yields

$$
\frac{\partial M}{\partial \sigma}=\ln \left(\frac{V^{*}}{V_{0}}\right)+\left(\alpha-\frac{\sigma^{2}}{2}\right)\left[\frac{1}{V^{*}} \frac{\partial^{2} V^{*}}{\partial \sigma^{2}}-\left(\frac{1}{V^{*}} \frac{\partial V^{*}}{\partial \sigma}\right)^{2}\right]
$$

Differentiating Eq. (14) with respect to $\sigma$ yields

$$
\frac{\partial^{2} V^{*}}{\partial \sigma^{2}}=\frac{2 I}{(\beta-1)^{3}}\left(\frac{\partial \beta}{\partial \sigma}\right)^{2}-\frac{I}{(\beta-1)^{2}} \frac{\partial^{2} \beta}{\partial \sigma^{2}}
$$

Substituting Eqs. (11), (14), and (A.10) into Eq. (A.9) yields

$$
\frac{\partial M}{\partial \sigma}=\ln \left(\frac{V^{*}}{V_{0}}\right)+\frac{\alpha-\sigma^{2} / 2}{\beta^{2}(\beta-1)^{2}}\left[(2 \beta-1)\left(\frac{\partial \beta}{\partial \sigma}\right)^{2}-\beta(\beta-1) \frac{\partial^{2} \beta}{\partial \sigma^{2}}\right] .
$$

Differentiating Eq. (13) with respect to $\sigma$ yields

$$
\begin{aligned}
\frac{\partial^{2} \beta}{\partial \sigma^{2}}= & \frac{2 \beta[\lambda \rho-\sigma(\beta-1)][\lambda \rho-\sigma(2 \beta-1)]}{\left[\sigma^{2}(\beta-1 / 2)+\alpha-\lambda \rho \sigma\right]^{2}} \\
& -\frac{\beta(\beta-1)}{\sigma^{2}(\beta-1 / 2)+\alpha-\lambda \rho \sigma}-\frac{\beta^{2} \sigma^{2}[\lambda \rho-\sigma(\beta-1)]^{2}}{\left[\sigma^{2}(\beta-1 / 2)+\alpha-\lambda \rho \sigma\right]^{3}} .
\end{aligned}
$$


Substituting Eqs. (13) and (A.12) into Eq. (A.11) yields

$$
\begin{aligned}
\frac{\partial M}{\partial \sigma}= & \ln \left(\frac{V^{*}}{V_{0}}\right)+\frac{\alpha-\sigma^{2} / 2}{(\beta-1)^{2}}\left\{\frac{[\lambda \rho-\sigma(\beta-1)]^{2}}{\left[\sigma^{2}(\beta-1 / 2)+\alpha-\lambda \rho \sigma\right]^{2}}+\frac{2 \beta(\beta-1) \sigma[\lambda \rho-\sigma(\beta-1)]}{\left[\sigma^{2}(\beta-1 / 2)+\alpha-\lambda \rho \sigma\right]^{2}}\right. \\
& \left.+\frac{(\beta-1)^{2}}{\sigma^{2}(\beta-1 / 2)+\alpha-\lambda \rho \sigma}+\frac{\beta(\beta-1) \sigma^{2}[\lambda \rho-\sigma(\beta-1)]^{2}}{\left[\sigma^{2}(\beta-1 / 2)+\alpha-\lambda \rho \sigma\right]^{3}}\right\},
\end{aligned}
$$

which is unambiguously positive since $\sigma(\beta-1)-\lambda \rho<0$ for all $\sigma \in\left(0, \sigma^{*}\right)$. It then follows from Eq. (23) that $\partial \mathrm{E}(T) / \partial \sigma$ is strictly increasing in $\sigma$ for all $\sigma \in\left(0, \sigma^{*}\right)$.

Substituting Eqs. (13) and (A.4) into Eq. (23) yields

$$
\frac{\partial \mathrm{E}(T)}{\partial \sigma}=\frac{\sigma \ln \left(V^{*} / V_{0}\right)}{\left(\alpha-\sigma^{2} / 2\right)^{2}}+\frac{\sigma(\beta-1)-\lambda \rho}{\left(\alpha-\sigma^{2} / 2\right)(\beta-1) \sqrt{\left(\sigma^{2} / 2-\alpha+\lambda \rho \sigma\right)^{2}+2 r \sigma^{2}}}
$$

It is evident from Eq. (9) that $\beta \rightarrow r / \alpha$ as $\sigma \rightarrow 0$. Thus, taking limit on both sides of Eq. (A.14) as $\sigma \rightarrow 0$ yields

$$
\lim _{\sigma \rightarrow 0} \frac{\partial \mathrm{E}(T)}{\partial \sigma}=-\frac{\lambda \rho}{\alpha(r-\alpha)}<0
$$

Since $\partial \mathrm{E}(T) / \partial \sigma$ is strictly increasing in $\sigma$ for all $\sigma \in\left(0, \sigma^{*}\right)$ and $\partial \mathrm{E}(T) / \partial \sigma>0$ for all $\sigma \in\left[\sigma^{*}, \sqrt{2 \alpha}\right)$, we conclude from Eq. (A.15) that there exists a unique point, $\sigma^{\circ} \in\left(0, \sigma^{*}\right)$, implicitly defined in Eq. (24), such that $\partial \mathrm{E}(T) / \partial \sigma<0$ for all $\sigma \in\left(0, \sigma^{\circ}\right)$ and $\partial \mathrm{E}(T) / \partial \sigma>0$ for all $\sigma \in\left(\sigma^{\circ}, \sqrt{2 \alpha}\right)$.

\section{Appendix D. Proof of Proposition 3}

Totally differentiating Eq. (24) with respect to $\alpha$ yields

$$
\frac{\mathrm{d} \sigma^{\mathrm{o}}}{\mathrm{d} \alpha}=-\left.\frac{\partial M}{\partial \alpha}\right|_{\sigma=\sigma^{\circ}} /\left.\frac{\partial M}{\partial \sigma}\right|_{\sigma=\sigma^{\circ}}
$$

From Eq. (A.13), we know that $\partial M / \partial \sigma>0$ for all $\sigma \in\left(0, \sigma^{*}\right)$ and in particular that $\partial M / \partial \sigma>0$ at $\sigma=\sigma^{\circ}$. Eq. (A.16) then implies that the sign of $\mathrm{d} \sigma^{\circ} / \mathrm{d} \alpha$ is opposite to 
that of $\partial M / \partial \alpha$ evaluated at $\sigma=\sigma^{\circ}$. To prove this proposition, it suffices to show that $\partial M / \partial \alpha<0$ at $\sigma=\sigma^{\mathrm{o}}$ if condition (26) holds.

Differentiating Eq. (A.8) with respect to $\alpha$ yields

$$
\frac{\partial M}{\partial \alpha}=\frac{1}{V^{*}}\left[\sigma \frac{\partial V^{*}}{\partial \alpha}+\frac{\partial V^{*}}{\partial \sigma}+\left(\alpha-\frac{\sigma^{2}}{2}\right)\left(\frac{\partial^{2} V^{*}}{\partial \sigma \partial \alpha}-\frac{1}{V^{*}} \frac{\partial V^{*}}{\partial \sigma} \frac{\partial V^{*}}{\partial \alpha}\right)\right]
$$

Differentiating Eq. (9) with respect to $\alpha$ yields

$$
\frac{\partial \beta}{\partial \alpha}=-\frac{\beta}{\sigma^{2}(\beta-1 / 2)+\alpha-\lambda \rho \sigma}
$$

Differentiating Eq. (13) with respect to $\alpha$ yields

$$
\frac{\partial^{2} \beta}{\partial \sigma \partial \alpha}=\frac{\beta[\sigma(3 \beta-2)-2 \lambda \rho]}{\left[\sigma^{2}(\beta-1 / 2)+\alpha-\lambda \rho \sigma\right]^{2}}+\frac{\beta^{2} \sigma^{2}[\lambda \rho-\sigma(\beta-1)]}{\left[\sigma^{2}(\beta-1 / 2)+\alpha-\lambda \rho \sigma\right]^{3}},
$$

where we have used Eq. (A.18). Differentiating Eq. (11) with respect to $\alpha$ yields

$$
\frac{\partial V^{*}}{\partial \alpha}=-\frac{I}{(\beta-1)^{2}} \frac{\partial \beta}{\partial \alpha}=\frac{V^{*}}{(\beta-1)\left[\sigma^{2}(\beta-1 / 2)+\alpha-\lambda \rho \sigma\right]}
$$

where the second equality follows from Eqs. (11) and (A.18). Differentiating Eq. (14) with respect to $\alpha$ yields

$$
\begin{aligned}
\frac{\partial^{2} V^{*}}{\partial \sigma \partial \alpha}= & \frac{2 I}{(\beta-1)^{3}} \frac{\partial \beta}{\partial \sigma} \frac{\partial \beta}{\partial \alpha}-\frac{I}{(\beta-1)^{2}} \frac{\partial^{2} \beta}{\partial \sigma \partial \alpha} \\
= & \frac{V^{*} \beta \sigma^{2}[\sigma(\beta-1)-\lambda \rho]}{(\beta-1)\left[\sigma^{2}(\beta-1 / 2)+\alpha-\lambda \rho \sigma\right]^{3}} \\
& -\frac{V^{*}[\sigma(\beta-1)(\beta-2)+2 \lambda \rho]}{(\beta-1)^{2}\left[\sigma^{2}(\beta-1 / 2)+\alpha-\lambda \rho \sigma\right]^{2}}
\end{aligned}
$$

where the second equality follows from Eqs. (11), (13), (A.18), and (A.19).

Using Eq. (10), we have

$$
\sigma \beta-\lambda \rho=\sqrt{\left(\frac{\sigma}{2}-\frac{\alpha-\lambda \rho \sigma}{\sigma}\right)^{2}+2 r}-\frac{2 \alpha-\sigma^{2}}{2 \sigma} .
$$


Note that

$$
\begin{aligned}
& {\left[\sqrt{\left(\frac{\sigma}{2}-\frac{\alpha-\lambda \rho \sigma}{\sigma}\right)^{2}+2 r}-\frac{2 \alpha-\sigma^{2}}{2 \sigma}\right]\left[\sqrt{\left(\frac{\sigma}{2}-\frac{\alpha-\lambda \rho \sigma}{\sigma}\right)^{2}+2 r}+\frac{2 \alpha-\sigma^{2}}{2 \sigma}\right]} \\
& =\left(\frac{\sigma}{2}-\frac{\alpha-\lambda \rho \sigma}{\sigma}\right)^{2}+2 r-\left(\frac{2 \alpha-\sigma^{2}}{2 \sigma}\right)^{2} \\
& =\frac{1}{\sigma}\left[\lambda \rho \sigma^{2}+\left(2 r+\lambda^{2} \rho^{2}\right) \sigma-2 \alpha \lambda \rho\right] .
\end{aligned}
$$

Inspection of Eqs. (A.22) and (A.23), we have $\sigma \beta-\lambda \rho<(>) 0$ if, and only if, $\sigma<(>) \sigma^{\diamond}$, where $\sigma^{\diamond}$ is given by

$$
\sigma^{\diamond}=\sqrt{\left(\frac{2 r+\lambda^{2} \rho^{2}}{2 \lambda \rho}\right)^{2}+2 \alpha}-\frac{2 r+\lambda^{2} \rho^{2}}{2 \lambda \rho} .
$$

Since $\sigma^{*} \beta-\lambda \rho=\sigma^{*}>0$, we have $\sigma^{*}>\sigma^{\diamond}$. Evaluating Eq. (23) at $\sigma=\sigma^{\diamond}$ yields

$$
\left.\frac{\partial \mathrm{E}(T)}{\partial \sigma}\right|_{\sigma=\sigma^{\diamond}}=\frac{\sigma^{\diamond}}{\left(\alpha-\sigma^{\diamond 2} / 2\right)^{2}}\left[\ln \left(\frac{\beta}{\beta-1}\right)+\ln \left(\frac{I}{V_{0}}\right)-\frac{1}{\beta-1}\right],
$$

where we have used Eqs. (11) and (14) and the fact that $\sigma^{\diamond} \beta-\lambda \rho=0$. Note that

$$
\frac{\partial}{\partial \beta}\left[\ln \left(\frac{\beta}{\beta-1}\right)+\ln \left(\frac{I}{V_{0}}\right)-\frac{1}{\beta-1}\right]=\frac{1}{\beta(\beta-1)^{2}}>0
$$

From Eq. (13), we have $\partial \beta / \partial \sigma>0$ for all $\sigma \in\left(0, \sigma^{*}\right)$. As $\sigma \rightarrow 0$, Eq. (9) implies that $\beta \rightarrow r / \alpha$. Thus, at $\sigma=\sigma^{\diamond}$, we have $\beta>r / \alpha$. Condition (26) and Eqs. (A.25) and (A.26) then imply that

$$
\left.\frac{\partial \mathrm{E}(T)}{\partial \sigma}\right|_{\sigma=\sigma^{\diamond}}>0
$$

Since $\partial \mathrm{E}(T) / \partial \sigma$ is strictly increasing in $\sigma$ for all $\sigma \in\left(0, \sigma^{*}\right)$, it follows from Eq. (24) and inequality (A.27) that $\sigma^{\circ}<\sigma^{\diamond}$. Thus, we have $\sigma^{\circ} \beta-\lambda \rho<0$.

Substituting Eqs. (14), (A.20), and (A.21) into Eq. (A.17) yields

$$
\frac{\partial M}{\partial \alpha}=\frac{\sigma \beta-\lambda \rho}{(\beta-1)\left[\sigma^{2}(\beta-1 / 2)+\alpha-\lambda \rho \sigma\right]}
$$




$$
\begin{aligned}
& +\left(\alpha-\frac{\sigma^{2}}{2}\right)\left\{\frac{\beta \sigma^{2}[\sigma(\beta-1)-\lambda \rho]}{(\beta-1)\left[\sigma^{2}(\beta-1 / 2)+\alpha-\lambda \rho \sigma\right]^{3}}\right. \\
& \left.-\frac{\sigma(\beta-1)^{2}+\lambda \rho}{(\beta-1)^{2}\left[\sigma^{2}(\beta-1 / 2)+\alpha-\lambda \rho \sigma\right]^{2}}\right\} .
\end{aligned}
$$

Since $\sigma^{\circ}<\sigma^{\diamond}<\sigma^{*}$, we have $\sigma^{\circ}(\beta-1)-\lambda \rho<\sigma^{\circ} \beta-\lambda \rho<0$. Thus, Eq. (A.28) implies that $\partial M / \partial \alpha<0$ at $\sigma=\sigma^{\circ}$ so that $\mathrm{d} \sigma^{\circ} / \mathrm{d} \alpha>0$.

\section{References}

Caballero, R.J., 1991. On the sign of the investment-uncertainty relationship. American Economic Review 81, 279-288.

Cappuccio, N., Moretto, M. 2001. Comments on the investment-uncertainty relationship in a real options model. Working paper, Department of Economics, University of Padova, Italy.

Dixit, A.K., 1993. The Art of Smooth Pasting. Harwood Academic Publishers, Chur, Switzerland.

Dixit, A.K., Pindyck, R.S., 1994. Investment under Uncertainty. Princeton University Press, Princeton, NJ.

Dumas, B., 1991. Super contact and related optimality conditions. Journal of Economic Dynamics and Control 4, 675-685.

Grenadier, S.R., Wang, N., 2005. Investment timing, agency, and information. Journal of Financial Economics 75, 493-533.

Karlin, S., Taylor, H.M., 1975. A First Course in Stochastic Processes. Academic Press, New York, NJ.

Leahy, J.V., Whited, T.M., 1996. The effect of uncertainty on investment: Some stylized facts. Journal of Money, Credit, and Banking 28, 64-83. 
Lund, D., 2005. How to analyze the investment-uncertainty relationship in real options models? Review of Financial Economics 14, 311-322.

McDonald, R.L., Siegel, D., 1986. The value of waiting to invest. Quarterly Journal of Economics 101, 707-727.

Merton, R.C., 1973a. An intertemporal capital asset pricing model. Econometrica 41, $867-887$.

Merton, R.C., 1973b. Theory of rational option pricing. Bell Journal of Economics and Management Science 4, 141-183.

Sarkar, S., 2000. On the investment-uncertainty relationship in a real options model. Journal of Economic Dynamics and Control 24, 219-225.

Shackleton, M.B., Sødal, S., 2005. Smooth pasting as rate of return equalization. Economics Letters 89, 200-206.

Shackleton, M.B., Wojakowski, R., 2002. The expected return and exercise time of Mertonstyle real options. Journal of Business, Finance and Accounting 29, 541-555. 